\title{
Criss-cross gait
}

\section{A clue to glucose transporter type 1 deficiency syndrome}

Francesca Magrinelli, MD, Eoin Mulroy, MD, FRACP, Susanne A. Schneider, MD, PhD, Anna Latorre, MD, PhD, Giulia Di Lazzaro, MD, Anita Hennig, MD, Stephanie Grünewald, MD, PhD, Darryl C. De Vivo, MD, and Kailash P. Bhatia, MD, DM, FRCP

Neurology ${ }^{\circledR}$ 2020;95:500-501. doi:10.1212/WNL.0000000000010502

Figure Overview of the pathogenesis, phenotypes, diagnosis, and treatment of glucose transporter type 1 deficiency syndrome (Glut1 DS)
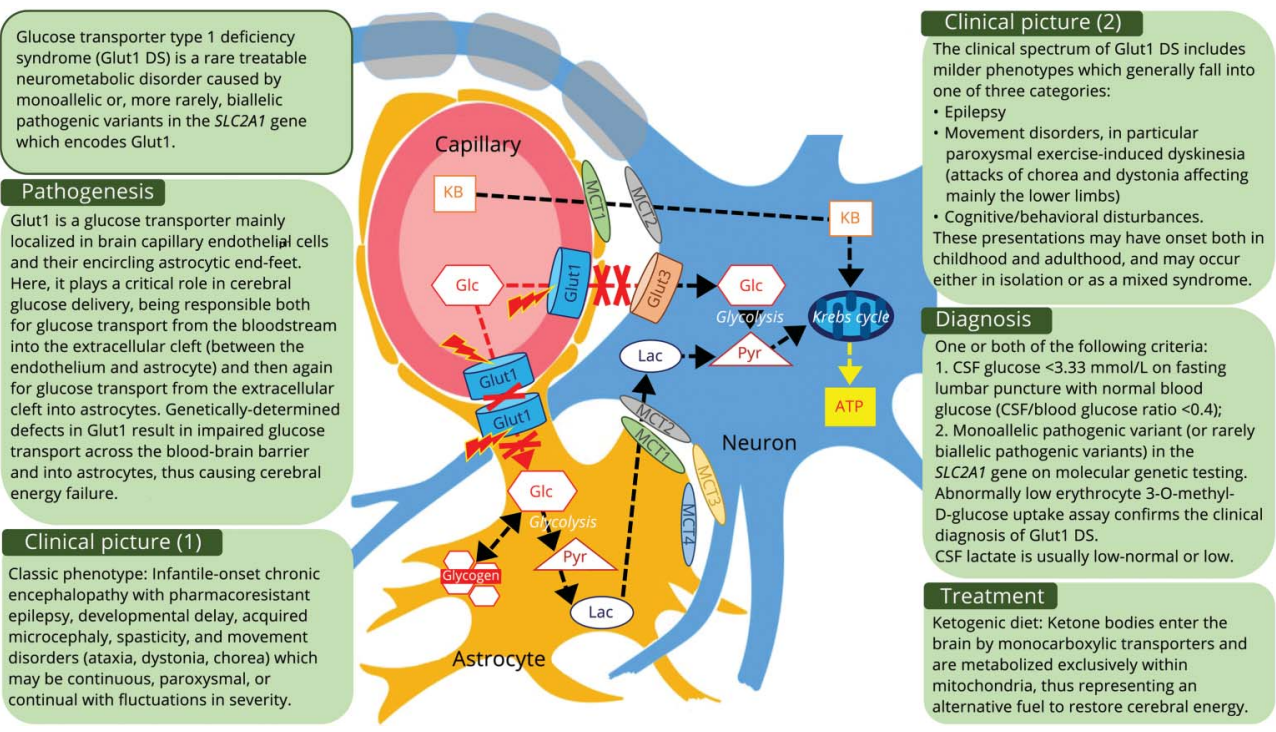

Red dashed lines indicate defective pathways in Glut1 DS. ATP = adenosine triphosphate; Glc = glucose; Glut = glucose transporter; $\mathrm{KB}=$ ketone bodies; $\mathrm{Lac}=$ lactate; $\mathrm{MCT}=$ monocarboxylic transporter; Pyr = pyruvate.

Nearly $90 \%$ of patients with glucose transporter type 1 deficiency syndrome (Glut1 DS; figure) have paroxysmal or constant gait abnormalities, including ataxic, spastic, ataxic-spastic, and dystonic gait. ${ }^{1,2}$ We report 3 cases of genetically proven Glut1 DS (table) demonstrating a distinctive paroxysmal gait disorder triggered by exertion or fasting, herein named "criss-cross gait" (video 1). It is characterized by lower-body choreo-dyskinesia causing the legs to intersect repeatedly, producing irregular, random steps combined with some loss of balance. Compensatory upper-body movements help maintain balance. In the appropriate clinical context, criss-cross gait should prompt evaluation for the treatable Glut1 DS and not be misinterpreted as functional.

\section{Acknowledgment}

The authors thank Kristin Engelstad, MS, CGC, Columbia University Irving Medical Center, New York, NY, for cooperation and all the patients who participated in the study.

\section{Correspondence}

Prof. Bhatia

k.bhatia@ucl.ac.uk
MORE ONLINE

\section{- Video}

From the Department of Clinical and Movement Neurosciences (F.M., E.M., A.L., G.D.L., K.P.B.), UCL Queen Square Institute of Neurology, University College London, UK; Department of Neurosciences, Biomedicine and Movement Sciences (F.M.), University of Verona, Italy; Department of Neurology (S.A.S., A.H.), Ludwig-Maximilians-Universität München, Germany; Department of Systems Medicine (G.D.L.), University of Roma Tor Vergata, Rome, Italy; Department of Metabolic Medicine (S.G.), UCL Great Ormond Street Hospital Institute of Child Health, NIHR Biomedical Research Center, London, UK; and Departments of Neurology and Pediatrics (D.C.D.V.), Columbia University Irving Medical Center, New York, NY.

Go to Neurology.org/N for full disclosures. Funding information and disclosures deemed relevant by the authors, if any, are provided at the end of the article. 
Table Clinical and genetic features of 3 patients with genetically confirmed glucose transporter type 1 deficiency syndrome showing the criss-cross gait (video 1)

\begin{tabular}{|c|c|c|c|}
\hline & Case 1 & Case 2 & Case 3 \\
\hline Current age, y & 54 & 25 & 24 \\
\hline Sex & Female & Male & Female \\
\hline Ethnicity & White British & White British & Germanic \\
\hline Age at onset & $6 y$ & $5 y$ & $11 \mathrm{mo}$ \\
\hline Clinical picture & $\begin{array}{l}\text { PED (episodes of toe curling, foot } \\
\text { dystonia, limb choreoathetosis) }\end{array}$ & $\begin{array}{l}\text { PED (episodes of foot dystonia, jerky } \\
\text { choreiform movements in limbs), } \\
\text { episodes of slurred speech }\end{array}$ & $\begin{array}{l}\text { Motor development delay, atypical } \\
\text { absence epilepsy, PED (episodes of } \\
\text { "wobbly gait"), mild intellectual disability }\end{array}$ \\
\hline Family history & $\begin{array}{l}\text { Father: history of paroxysmal dystonic } \\
\text { choreoathetosis, possibly affected } \\
\text { (retrospectively); son affected }\end{array}$ & Father affected & Negative (de novo mutation) \\
\hline CSF analysis & Not performed & $\begin{array}{l}\text { CSF glucose }=1.9 \mathrm{mmol} / \mathrm{L} \text { (with blood } \\
\text { glucose }=6.8 \mathrm{mmol} / \mathrm{L}), \mathrm{CSF} / \text { blood glucose } \\
\text { ratio }=0.28\end{array}$ & Not available \\
\hline $\begin{array}{l}\text { Genetic testing } \\
\text { SLC2A1 } \\
\text { (ENST00000426263) }\end{array}$ & $\begin{array}{l}\text { Heterozygous variant c.601T>C } \\
\text { (p.Cys201Arg) }\end{array}$ & $\begin{array}{l}\text { Heterozygous variant c. } 278 \mathrm{G}>\mathrm{A} \\
\text { (p.Arg93Gln) }\end{array}$ & $\begin{array}{l}\text { Heterozygous variant c.998G>A } \\
\text { (p.Arg333GIn) }\end{array}$ \\
\hline $\begin{array}{l}\text { Treatment and } \\
\text { follow-up }\end{array}$ & $\begin{array}{l}\text { Patient declined to start ketogenic diet; } \\
\text { overall reduction in the intensity and } \\
\text { frequency of paroxysmal symptoms } \\
\text { with age over a 20-year follow-up }\end{array}$ & $\begin{array}{l}\text { Ketogenic diet since age } 6 \text { with marked } \\
\text { improvement of symptoms; episodes } \\
\text { characterized by mild twitching in feet, } \\
\text { difficulty concentrating, and slurred } \\
\text { speech may occur in relation to } \\
\text { occasional dietary indiscretions }\end{array}$ & $\begin{array}{l}\text { Ketogenic diet since age } 12 \text { with low } \\
\text { adherence; mild constant gait } \\
\text { unsteadiness and occasional paroxysmal } \\
\text { worsening of gait disturbance over a 12- } \\
\text { year follow-up }\end{array}$ \\
\hline
\end{tabular}

Abbreviation: PED = paroxysmal exercise-induced dyskinesia.

\section{Study funding}

No targeted funding reported.

\section{Disclosure}

F. Magrinelli and G. Di Lazzaro are supported by the European Academy of Neurology (EAN) Research Fellowship 2020. All other authors report no disclosures relevant to the manuscript. Go to Neurology.org/N for full disclosures.

Appendix Authors

\begin{tabular}{|c|c|c|}
\hline Name & Location & Contribution \\
\hline $\begin{array}{l}\text { Francesca } \\
\text { Magrinelli, } \\
\text { MD }\end{array}$ & $\begin{array}{l}\text { UCL Queen Square } \\
\text { Institute of Neurology, } \\
\text { University College London, } \\
\text { UK }\end{array}$ & $\begin{array}{l}\text { Designed and } \\
\text { conceptualized study, } \\
\text { major role in the acquisition } \\
\text { of data, analyzed the data, } \\
\text { drafted the manuscript for } \\
\text { intellectual content }\end{array}$ \\
\hline $\begin{array}{l}\text { Eoin } \\
\text { Mulroy, MD, } \\
\text { FRACP }\end{array}$ & $\begin{array}{l}\text { UCL Queen Square } \\
\text { Institute of Neurology, } \\
\text { University College London, } \\
\text { UK }\end{array}$ & $\begin{array}{l}\text { Designed and } \\
\text { conceptualized study, major } \\
\text { role in the acquisition of } \\
\text { data, revised the manuscript } \\
\text { for intellectual content }\end{array}$ \\
\hline $\begin{array}{l}\text { Susanne A. } \\
\text { Schneider, } \\
\text { MD, PhD }\end{array}$ & $\begin{array}{l}\text { Ludwig-Maximilians- } \\
\text { Universität München, } \\
\text { Germany }\end{array}$ & $\begin{array}{l}\text { Major role in the acquisition } \\
\text { of data, revised the } \\
\text { manuscript for intellectual } \\
\text { content }\end{array}$ \\
\hline $\begin{array}{l}\text { Anna } \\
\text { Latorre, } \\
\text { MD, PhD }\end{array}$ & $\begin{array}{l}\text { UCL Queen Square } \\
\text { Institute of Neurology, } \\
\text { University College London, } \\
\text { UK }\end{array}$ & $\begin{array}{l}\text { Major role in the acquisition } \\
\text { of data, revised the } \\
\text { manuscript for intellectual } \\
\text { content }\end{array}$ \\
\hline
\end{tabular}

Appendix (continued)

\begin{tabular}{|c|c|c|}
\hline Name & Location & Contribution \\
\hline $\begin{array}{l}\text { Giulia Di } \\
\text { Lazzaro, MD }\end{array}$ & $\begin{array}{l}\text { UCL Queen Square } \\
\text { Institute of Neurology, } \\
\text { University College London, } \\
\text { UK }\end{array}$ & $\begin{array}{l}\text { Major role in the acquisition } \\
\text { of data, revised the } \\
\text { manuscript for intellectual } \\
\text { content }\end{array}$ \\
\hline $\begin{array}{l}\text { Anita } \\
\text { Hennig, MD }\end{array}$ & $\begin{array}{l}\text { Ludwig-Maximilians- } \\
\text { Universität München, } \\
\text { Germany }\end{array}$ & $\begin{array}{l}\text { Major role in the acquisition } \\
\text { of data, revised the } \\
\text { manuscript for intellectual } \\
\text { content }\end{array}$ \\
\hline $\begin{array}{l}\text { Stephanie } \\
\text { Grünewald, } \\
\text { MD, PhD }\end{array}$ & $\begin{array}{l}\text { UCL Great Ormond Street } \\
\text { Hospital Institute of Child } \\
\text { Health, NIHR Biomedical } \\
\text { Research Center, London, } \\
\text { UK }\end{array}$ & $\begin{array}{l}\text { Major role in the acquisition } \\
\text { of data, revised the } \\
\text { manuscript for intellectual } \\
\text { content }\end{array}$ \\
\hline $\begin{array}{l}\text { Darryl C. De } \\
\text { Vivo, MD }\end{array}$ & $\begin{array}{l}\text { Columbia University Irving } \\
\text { Medical Center, New York, } \\
\text { NY }\end{array}$ & $\begin{array}{l}\text { Major role in the acquisition } \\
\text { of data, revised the } \\
\text { manuscript for intellectual } \\
\text { content }\end{array}$ \\
\hline $\begin{array}{l}\text { Kailash P. } \\
\text { Bhatia, MD, } \\
\text { DM, FRCP }\end{array}$ & $\begin{array}{l}\text { UCL Queen Square } \\
\text { Institute of Neurology, } \\
\text { University College London, } \\
\text { UK }\end{array}$ & $\begin{array}{l}\text { Design and conceptualized } \\
\text { study, major role in the } \\
\text { acquisition of data, revised } \\
\text { the manuscript for } \\
\text { intellectual content }\end{array}$ \\
\hline
\end{tabular}

\section{References}

1. Wang D, Pascual JM, De Vivo D. Glucose transporter type 1 deficiency syndrome. In: Adam MP, Ardinger HH, Pagon RA, et al, eds. GeneReviews ${ }^{\circledR}$. Seattle: GeneReviews; 1993 [last update: March 1, 2018].

2. Pons R, Collins A, Rotstein M, Engelstad K, De Vivo DC. The spectrum of movement disorders in Glut-1 deficiency. Mov Disord 2010;25:275-281. 


\section{Neurology}

\section{Criss-cross gait: A clue to glucose transporter type 1 deficiency syndrome \\ Francesca Magrinelli, Eoin Mulroy, Susanne A. Schneider, et al. \\ Neurology 2020;95;500-501 Published Online before print August 4, 2020 \\ DOI 10.1212/WNL.0000000000010502}

This information is current as of August 4, 2020

\section{Updated Information \& Services}

References

Subspecialty Collections

Permissions \& Licensing

Reprints including high resolution figures, can be found at: http://n.neurology.org/content/95/11/500.full

This article cites 1 articles, 0 of which you can access for free at: http://n.neurology.org/content/95/11/500.full\#ref-list-1

This article, along with others on similar topics, appears in the following collection(s):

All Clinical Neurology

http://n.neurology.org/cgi/collection/all_clinical_neurology

All Genetics

http://n.neurology.org/cgi/collection/all_genetics

All Movement Disorders

http://n.neurology.org/cgi/collection/all_movement_disorders

Gait disorders/ataxia

http://n.neurology.org/cgi/collection/gait_disorders_ataxia

Metabolic disease (inherited)

http://n.neurology.org/cgi/collection/metabolic_disease_inherited

Information about reproducing this article in parts (figures,tables) or in its entirety can be found online at:

http://www.neurology.org/about/about_the_journal\#permissions

Information about ordering reprints can be found online:

http://n.neurology.org/subscribers/advertise

Neurology ${ }^{\circledR}$ is the official journal of the American Academy of Neurology. Published continuously since 1951, it is now a weekly with 48 issues per year. Copyright (O) 2020 American Academy of Neurology. All rights reserved. Print ISSN: 0028-3878. Online ISSN: 1526-632X.

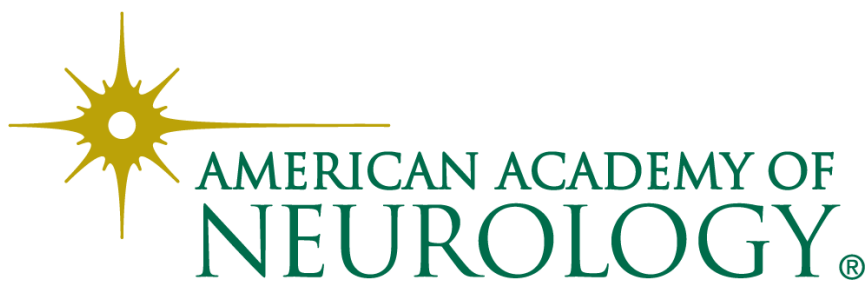

